\title{
Japanese encephalitis virus RNA detected in Culex pipiens mosquitoes in Italy
}

P Ravanini (paolo.ravanini@gmail.com) ${ }^{1}$, E Huhtamo ${ }^{2}$, V Ilaria ${ }^{1}$, M G Crobu ${ }^{1}$, A M Nicosia ${ }^{1}$, L Servino ${ }^{1}$, F Rivasi ${ }^{3}$, S Allegrini ${ }^{4}$, U Miglio ${ }^{4}$ A Magri ${ }^{5}$, R Minisini ${ }^{5}$, V Vapalahti $^{2}$, R Boldorini ${ }^{4}$

1. Laboratory of Molecular Virology, Azienda Ospedaliero-Universitaria Maggiore della Carità, Novara, Italy

2. Infection Biology Research Programme, Department of Virology, Haartman Institute, Faculty of Medicine, University of Helsinki, Helsinki, Finland

3. Department of Diagnostics, Laboratory Service and Forensic Medicine, Section of Pathological Anatomy, University of Modena and Reggio Emilia, Italy

4. Department of Pathological Anatomy, Faculty of Medicine and Surgery, University Amedeo Avogadro del Piemonte Orientale, Novara, Italy

5. Department of Translational Medicine, University of Eastern Piedmont 'Amedeo Avogadro', Novara, Italy

Citation style for this article:

Ravanini P, Huhtamo E, Ilaria V, Crobu MG, Nicosia AM, Servino L, Rivasi F, Allegrini S, Miglio U, Magri A, Minisini R, Vapalahti O, Boldorini R. Japanese encephalitis virus RNA detected in Culex pipiens mosquitoes in Italy. Euro Surveill. 2012;17(28):pii=20221. Available online: http://www.eurosurveillance.org/ViewArticle.

aspx?Articleld $=20221$

Article submitted on 4 July 2012 / published on 12 July 2012

Mosquitoes collected in northern Italy were screened for flavivirus RNA. Positive amplicons were sequenced and found most similar to insect flavivirus (ISF), Usutu virus (USUV) and surprisingly also to Japanese encephalitis virus (JEV). The sequence ( $167 \mathrm{bp})$, obtained from one pool of Culex pipiens, was found identical to JEV strains from bats in China. Unfortunately additional sequence data or virus isolations were not obtained in this study. Confirmation of potential introduction of JEV to Italy and other European countries is urgently needed.

In the course of a small-scale preliminary study screening for the presence of flavivirus RNA in mosquitoes in Italy, we obtained sequences of three different flaviviruses; an insect-specific flavivirus (ISF) related to cell fusing agent virus, Usutu virus (USUV) and, to our surprise, also of Japanese encephalitis virus (JEV). While ISF and USUV have been documented previously in Italy and several other European countries [1,2], JEV has, to our knowledge, not been detected in mosquitoes in Europe so far. JEV is a mosquito-borne flavivirus known to be endemic in Asia, extending to India and Pakistan in the west, where it is a leading cause of encephalitis. Although commercial inactivated vaccines are available against JEV, it causes an estimated annual number of 30,000-50,000 cases worldwide [3]. The majority of the infections are subclinical, but up to $30 \%$ of symptomatic patients die, and $30 \%$ of the survivors have persistent neurological sequelae [3]. The life cycle of JEV includes Culex spp. mosquitoes and water birds or pigs, but JEV also infects a wide range of other vertebrates. In addition to humans, horses may develop encephalitis and are considered dead-end hosts for JEV transmission [4].

\section{Sample collection}

Following the active circulation of WNV and USUV, the recent detection of novel ISFs in Italy and elsewhere in Europe, and the detection of dengue virus in southern France and Croatia $[1,7,10,11]$, the aim of this study was to screen mosquitoes for flavivirus RNA using a system allowing the detection of all flaviviruses. Female mosquitoes were collected in late summer of 2010 and 2011 in rural areas near Modena and Bologna in EmiliaRomagna region (Figure 1 ), using $\mathrm{CO}_{2}$-baited traps.

Mosquitoes were identified using morphological characteristics [5], pooled by species (identification at subspecies level was not done), date and site of collection (with a maximum of 27 individuals per pool) and stored at $-80^{\circ} \mathrm{C}$ until processed. The mosquito species collected included mainly $C$. pipiens, and additionally Aedes albopictus, $A$. caspius and $A$. vexans. A total of 62 pools were studied; 52 had been collected in 2010 (all C. pipiens) and 10 in 2011 (five C. pipiens).

\section{Molecular analysis}

The mosquitoes were ground manually using sterile sand and Dulbecco's phosphate-buffered saline. Nucleic acids were extracted using EasyMag (bioMérieux) and examined by RT-PCR targeted to a conserved region of the flavivirus NS5 gene [6]. The PCR products were sequenced directly and cloned when necessary (CloneJET PCR Cloning Kit, Fermentas). The obtained sequences were identified using BLAST (blast.ncbi. nlm.nih.gov/Blast.cgi).

Of the 62 pools, five were found positive for flavivirus RNA. The sequences (Box) were identified as (i) ISF from $A$. albopictus, 2011 (two sequences; lengths 133 and $87 \mathrm{bp}$; identical in the overlapping region), (ii) USUV from C. pipiens, 2010 and 2011 (two sequences; lengths 133 and $167 \mathrm{bp}$; identical in the overlapping 


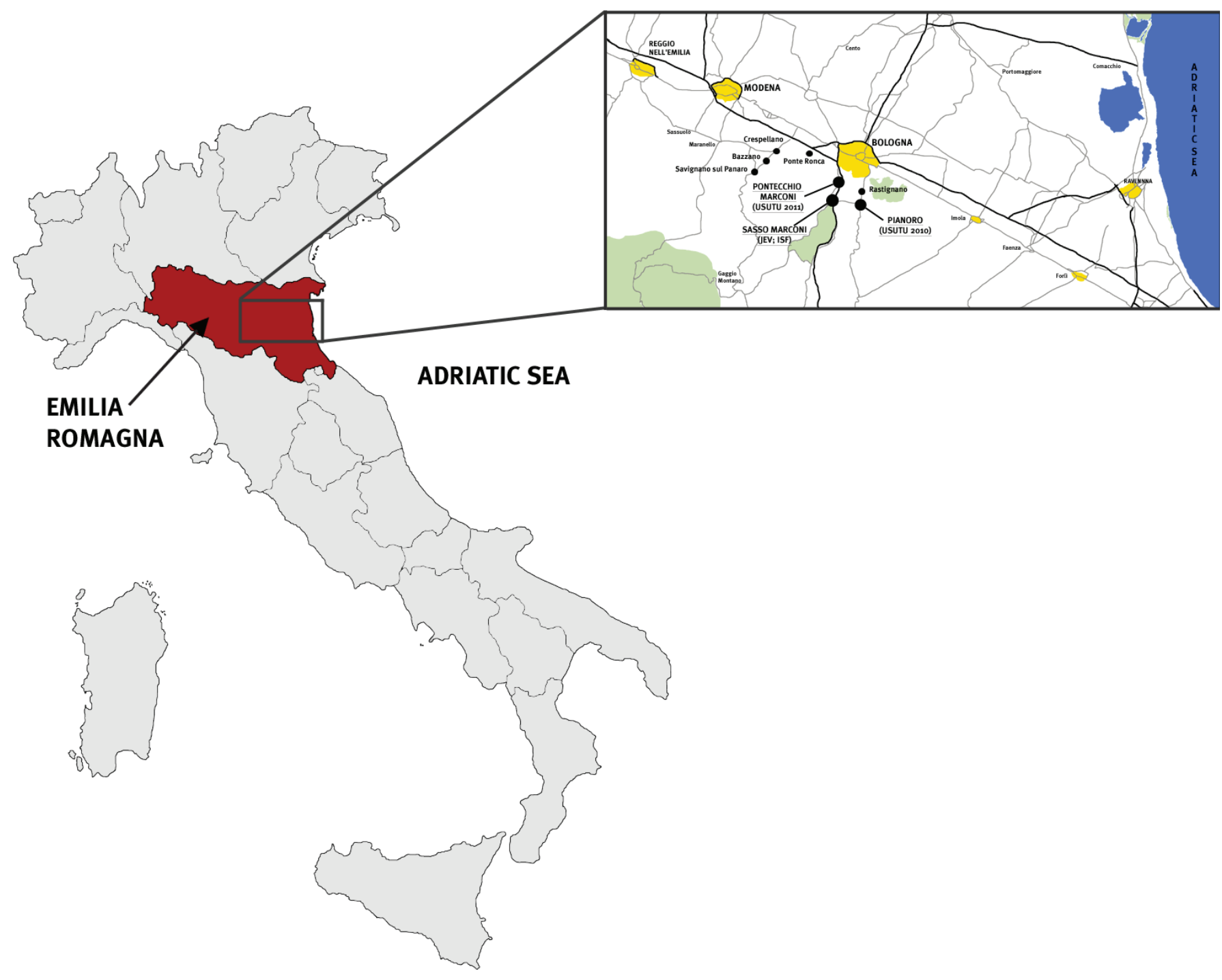

The black dots represent the eight collection sites near Bologna and Modena.

region) and (iii) JEV (one sequence; $167 \mathrm{bp}$ ) from one pool of C. pipiens, 2010 (Figure 2). The ISF (collected in Sasso Marconi) and USUV (collected in Pontecchio Marconi and Pianoro) sequences were identical to other sequences previously reported from Italy $[1,7]$. The JEV sequence (genomic position: 9,109-9,275) was obtained from mosquitoes collected in Sasso Marconi. It showed $100 \%$ similarity to four sequences in Genbank, all of them representing JEV genotype III viruses isolated from bats in China between 1986 and 2009 (JN711458, JN711459, JF706285, JF185036).

The PCR product yielding the $\mathrm{NS}_{5}$ sequence related to JEV was amplified from the original material twice and sequenced in three separate laboratories. Additional sequence data would be needed for detailed characterisation of the viral strain and sequence analysis, but unfortunately the attempts to amplify longer sequences from the JEV-positive pool using primers targeted to $\mathrm{E}, \mathrm{NS}_{5}$ and $\mathrm{NS}_{3}$ regions in nested and seminested protocols remained negative. Attempts to isolate the virus from the JEV- and USUV-positive pools on Vero and on $\mathrm{C} 6 / 36$ insect cells were not successful.

\section{Discussion}

While the potential risk of JEV spreading to Europe has been acknowledged before [8], and despite the active surveillance for flaviviruses such as WNV and USUV, to the best of our knowledge, this is the first report of a JEV-like sequence in mosquitoes in Europe. The JEV-like sequence was detected within a small scale preliminary study, and some details of the field work along 
Box

Viral nucleotide sequence fragments obtained from mosquitoes collected in Italy, summer 2010 and 2011 $(\mathrm{n}=3)$

JEV_pool_M20 167 bp

TCATGTGGCTTGGAGCACGGTATCTAGAGTTTGAAGCTTTGGGGTTCCT GAATGAAGACCATTGGCTGAGCCGAGAGAATTCAGGAGGTGGAGTGG AAGGCTCAGGCGTCCAAAAGCTAGGATACATCCTCCGTGACATAGCAGG AAAGCAAGGAGGGAAAATGTAC

USUV_pool_M7 167 bp

TCATGTGGCTAGGCGCCAGATTCCTGGAGTTTGAAGCTCTGGGCTTTCT GAATGAGGACCATTGGTTAGGAAGAAAGAATTCTGGAGGAGGTGTTG AAGGACTTGGTGTCCAAAAACTTGGTTACATTCTGCGTGAGATGAGCC ACCATTCAGGTGGGAAAATGTAC

ISF_pool_M2B 133 bp

CTCG GAAGTCGTTTTCTGGAATTTGAG GCCTTGGGGTTCCTAAAT GCTGATCACTGGGTCAGTCGTGAAAACTTTCCTGGGGGCGTGGGT GGAGTGGGTGTCAATTACTTTGGCAACTACCTAAAGGAAATTT

with mosquito subspecies identification were unfortunately not documented in detail. Laboratory contamination as the source of the obtained JEV sequence was highly unlikely, as no JEV virus, RNA or PCR products had ever been handled in the laboratories where the mosquitoes were processed or where the RT-PCRs were performed. Interestingly, Mani et al. have reported detecting in 1996-97 JEV antibodies and RNA in Italian birds [9], but unfortunately no further information is currently available about the sequences found in that study.

Recently, autochthonous dengue virus (DENV) infections have been detected in France [10] and Croatia [11]. While these viruses are most likely to have been imported there from endemic regions, most probably through viraemic travellers or via materials harbouring infected mosquitoes, eggs or larvae, JEV could have been introduced to Italy through waterfowl or wild waterbirds. Future arbovirus surveillance should include JEV-specific or pan-flavivirus detection methods, and it should be noted that due to cross-reactions, serological assays with the exception of seroneutralisation are probably unable to differentiate an immune response to JEV from one to WNV and USUV.

\section{Conclusions}

A partial genomic sequence of JEV was detected in Italian $C$. pipiens mosquitoes for the first time, but confirmation of the finding by additional sequence data or virus isolation has not yet been successful. The authors are aware that these findings are preliminary, and confirmation of the results is necessary. Further evidence of JEV circulation is required for evaluating the possible need for precautionary measures against JEV transmission in Italy and other European countries.
FIGURE 2

Phylogenetic tree based on a 122 bp region of flavivirus NS5 sequences obtained from mosquitoes collected in Italy, summer 2010 and $2011(n=3)$

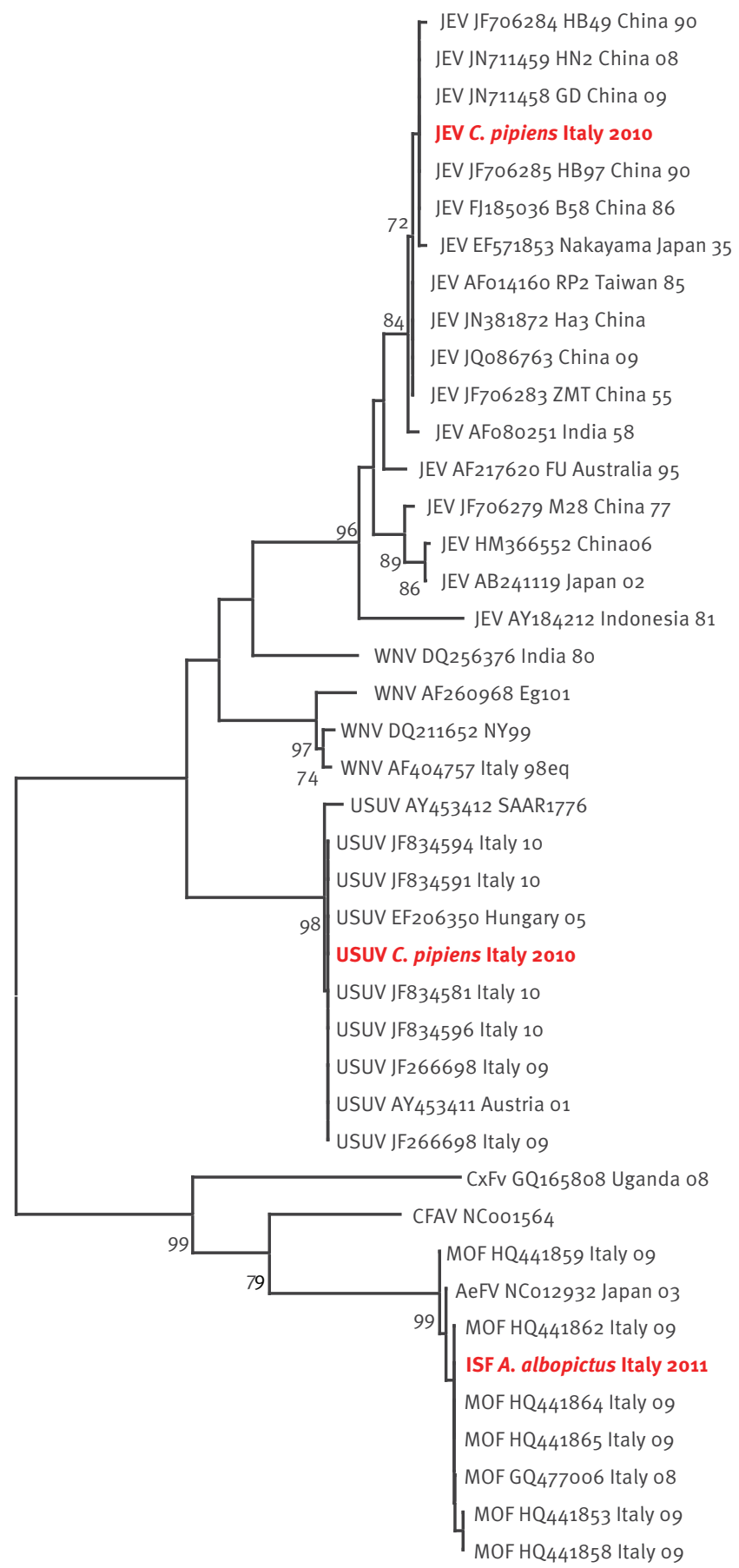

0.1

ISF: insect-specific flavivirus; JEV: Japanese encephalitis virus; USUV: Usutu virus.

Mosquito pools positive for JEV and ISF were collected at Sasso Marconi, and pools positive for USUV from Pontecchio Marconi and Pianoro. The neighbour-joining phylogenetic tree was estimated using maximum composite likelihood model, including 1,000 bootstrap replicates using programme Mega. 
We thank Alberto Palandri for the graphic support.

\section{References}

1. Calzolari M, Zé-Zé L, Ruzek D, Vázquez A, Jeffries C, Defilippo $\mathrm{F}$, et al. Detection of mosquito-only flaviviruses in Europe. J Gen Virol. 2012;93(Pt6):1215-25.

2. Vazquez A, Jimenez-Clavero M, Franco L, Donoso-Mantke 0 , Sambri V, Niedrig M, et al. Usutu virus - potential risk of human disease in Europe. Euro Surveill. 2011;16(31):pii=19935. Available from: http://www.eurosurveillance.org/ViewArticle. aspx?Articleld $=19935$

3. Centers for Disease Control and Prevention (CDC). Japanese encephalitis Factsheet. Atlanta: CDC. [Accessed 11 July 2012]. Available from: http://www.cdc.gov/ncidod/dvbid/ jencephalitis/facts.htm

4. Mackenzie JS, Gubler DJ, Petersen LR. Emerging flaviviruses: the spread and resurgence of Japanese encephalitis, West Nile and dengue viruses. Nat Med. 2004;10(12 Suppl):S98-109.

5. Schaffner E, Angel G, Geoffroy B, Hervy JP, Rhaiem A, Brunhes J. The Mosquitoes of Europe. CD-ROM. CD-ROM. Montpellier: Institut de Recherche pour le Développement/Entente interdépartementale pour la démoustication du littoral (EID) Méditerrannée ; 2001.

6. Scaramozzino N, Crance JM, Jouan A, DeBriel DA, Stoll F, Garin D. Comparison of flavivirus universal primer pairs and development of a rapid, highly sensitive heminested reverse transcription-PCR assay for detection of flaviviruses targeted to a conserved region of the $\mathrm{NS}_{5}$ gene sequences. J Clin Microbiol. 2001;39(5):1922-7.

7. Calzolari M, Gaibani P, Bellini R, Defilippo F, Pierro A, Albieri A, et al. Mosquito, Bird and Human Surveillance of West Nile and Usutu Viruses in Emilia-Romagna Region (Italy) in 2010. PLoS One. 2012;7(5):e38058.

8. Pfeffer M, Dobler G. Emergence of zoonotic arboviruses by animal trade and migration. Parasit Vectors. 2010;8;3(1):35.

9. Mani P, Legrottaglie R, Bertelloni F, Fratini F, Filogari D, Rossi G. Il Virus dell'Encefalite Giapponese (JEV) in uccelli selvatici sinantropici (Passer italiae, Turdus merula, Sturnus vulgaris) e da richiamo (Turdus ilacus) in Toscana. [The Japanese encephalitis virus (JEV) in synanthropic wild birds (Passer italiae, Turdus merula, Sturnus vulgaris) and redwing (Turdus ilacus) in Tuscany]. Ecologia Urbana. 2009;21(1);99-100. Italian.

10. La Ruche G, Souarès $Y$, Armengaud A, Peloux-Petiot F, Delaunay $P$, Desprès $P$, et al. First two autochthonous dengue virus infections in metropolitan France, September 2010. Euro Surveill. 2010;15(39):pii=19676. Available from: http://www. eurosurveillance.org

11. Schmidt-Chanasit J, Haditsch M, Schoneberg I, Gunther S, Stark K, Frank C. Dengue virus infection in a traveller returning from Croatia to Germany. Euro Surveill. 2010;15(40): pii=19677. Available from: http://www.eurosurveillance.org/ViewArticle. aspx?Articleld $=19677$. 